\title{
Patient With a Massive Aortic Arch Aneurysm With Contained Rupture
}

\author{
Bulat A. Ziganshin \\ Aortic Institute, Yale University School of Medicine, New Haven, Connecticut
}

\section{Key Words}

\section{Aortic arch aneurysm - Aneurysm rupture - Surgical treatment}

The patient is a 66-year-old African American male who presented to an outside emergency room in serious condition with neck pain as well as neck and facial swelling and difficulty swallowing of about 5 weeks duration. He finally came for attention when the pain became very severe. He was a chronically ill-appearing male bordering on cachexia. His evaluation at the referring hospital showed an ascending and arch aneurysm with rupture of the aortic arch and a large mediastinal hematoma (Fig. 1A). The patient had a significant history of hypertension, dyslipidemia, chronic obstructive pulmonary disease, and heavy smoking. He was transferred to our institution for further care.

The preoperative computed tomography (CT) showed a massive ruptured aneurysm of the aortic arch measuring up to $11.7 \mathrm{~cm}$ (Fig. 2).

On entry into the pericardium we discovered a contained rupture of what turned out to be a false aneurysm containing a clot that had breeched the aortic wall, resulting in a large cavity between the innominate and left carotid arteries. The patient was placed on bypass through the femoral vessels and an aortic valve replacement (severe aortic regurgitation on echocardiogram), ascending aortic replacement (root sparing), and formal arch replacement with stage 1 elephant trunk was performed. Deep hypothermic circulatory arrest $\left(31\right.$ minutes at $18^{\circ} \mathrm{C}$ ) and

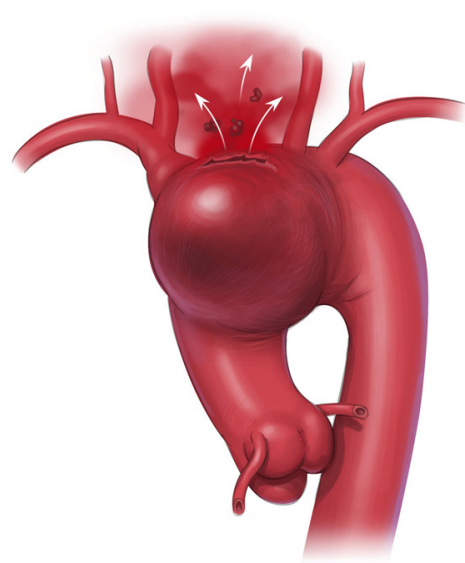

A

Figure 1. (A) Illustrates the appearance of the aortic arch rupture discovered intraoperatively. (B) Shows the complete reconstruction of the ascending aorta and aortic arch.

bilateral antegrade cerebral perfusion (14 minutes at $400 \mathrm{cc} /$ minute) was required for cerebral protection. The formal arch replacement included anastomosing the innominate artery and the left carotid artery endto-end between each other with a 12-mm graft, which was then anastomosed with the main arch graft with a separate $10-\mathrm{mm}$ graft end-to-side of both grafts (see Fig. 1B). The total bypass time and cross-clamp times were 214 and 163 minutes, respectively.

The patient had a difficult postoperative recovery, requiring reintubation. The patient also developed atrial fibrillation, which resolved before discharge. Five months postoperatively the patient is doing well.

Corresponding author:

Bulat A. Ziganshin

Aortic Institute, Yale University School of Medicine

Boardman 204

330 Cedar St.

New Haven, CT 06510 (USA)

Tel. +1 203785 2551, Fax +1 203785 3346, E-Mail: bulat.ziganshin@yale.edu 


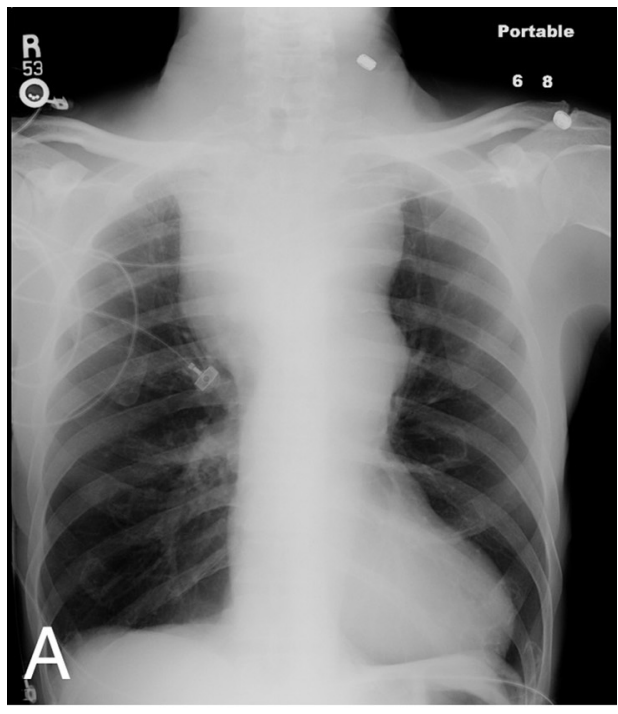

This case is especially interesting for the extraordinary preoperative images of the aortic arch (Fig. 2). We encourage similar submissions to the AORTA journal's category "Images in Aortic Disease."

\section{Comment on this Article or Ask a Question}

Cite this article as: Ziganshin BA. Patient With a Massive Aortic Arch Aneurysm With Contained Rupture. Aorta 2013;1(1): 57-58. DOI: http://dx.doi.org/10.12945/j.aorta.2013.13.019
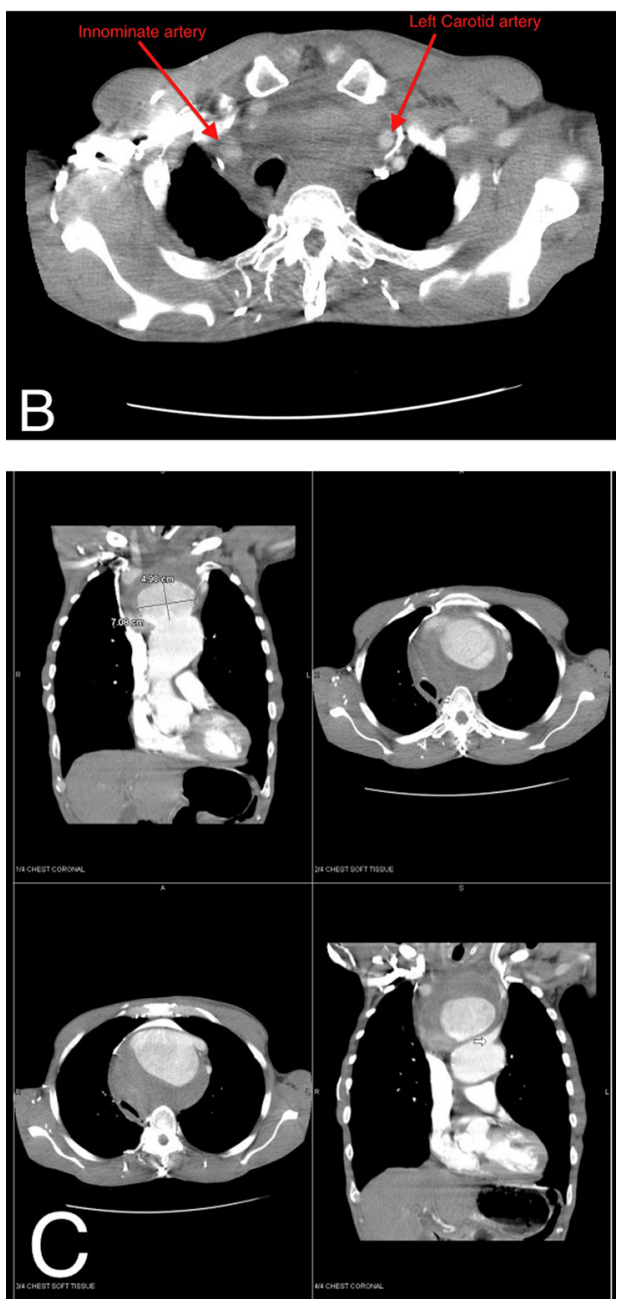

Figure 2. Preoperative chest X-ray (A) and CT scan images (B) and $(C)$ showing a massive aneurysm of the aortic arch. On the axial CT image please note how far apart the innominate and left carotid arteries are. 